\title{
VALOR DE LA DENSIDAD DEL ANTÍGENO PROSTÁTICO ESPECÍFICO Y DE LA DENSIDAD DEL ANTÍGENO PROSTÁTICO ESPECÍFICO DE LA ZONA TRANSICIONAL EN EL DIAGNÓSTICO DEL CÁNCER DE PRÓSTATA
}

\author{
J.M. MARTÍNEZ JABALOYAS, F. GARCÍA MORATA, R. VILLAMÓN FORT, \\ F. PASTOR HERNÁNDEZ, M. GIL SALOM, F. GARCÍA SISAMÓN
}

Servicio de Urología. Hospital Clínico Universitario de Valencia. Valencia.

Actas Urol Esp. 27 (6): 442-449, 2003

\section{RESUMEN}

VALOR DE LA DENSIDAD DEL ANTÍGENO PROSTÁTICO ESPECÍFICO Y DE LA DENSIDAD DEL ANTÍGENO PROSTÁTICO ESPECÍFICO DE LA ZONA TRANSICIONAL EN EL DIAGNÓSTICO DEL CÁNCER DE PRÓSTATA

INTRODUCCIÓN: Cuando el antígeno prostático específico (PSA) sérico se encuentra entre 4,1 y $10 \mathrm{ng} / \mathrm{ml}$ un número importante de pacientes son sometidos a biopsia de forma innecesaria. Intentamos determinar si el cálculo de la densidad de PSA (DPSA) y la densidad de PSA de la zona de transición (DPSAZT) mejoran la capacidad del PSA para discriminar entre enfermedad benigna y cáncer.

MÉTODOS: Estudio prospectivo en el que se incluyeron 314 varones con cifras de PSA entre 4,1 y $10 \mathrm{ng} / \mathrm{ml}$. En todos ellos se realizó ecografia transrectal y biopsia prostática, calculando el volumen prostático y de la zona de transición. Se calculó la DPSA y la DPSAZT para cada paciente. Se realizaron curvas de rendimiento diagnóstico (ROC curves) para PSA, DPSA y DPSAZT para la totalidad de los pacientes y seleccionando aquellos con tacto rectal no sospechoso de malignidad. Se calculó sensibilidad y especificidad para diferentes puntos de corte para el DPSA y DPSAZT.

RESULTADOS: Para la totalidad de la muestra, tanto DPSA como DPSAZT mostraron un área bajo la curva mayor que PSA (p<0,05), sin que se apreciasen diferencias entre DPSAZT y DPSA. El punto de corte con mayor eficiencia diagnóstica para DPSA fue de $0,17 \mathrm{ng} / \mathrm{ml} / \mathrm{cc}$ (sensibilidad de $71,4 \%$ y especificidad de $55,7 \%$ ) y para DPSAZT de $0,41 \mathrm{ng} / \mathrm{ml} / \mathrm{cc}$ (sensibilidad $70 \%$ y especificidad de $61,5 \%$ ). Cuando el tacto rectal era normal no se observaron diferencias entre PSA y DPSA y sí entre PSA y DPSAZT. En cualquier modo el área bajo la curva o exactitud fue siempre inferior al 70\% y para evitar un número considerable de biopsias (alta especificidad) se dejan por diagnosticar un número considerable de cánceres (baja sensibilidad).

CONCLUSIONES: Consideramos que la DPSA y DPSAZT no son métodos excesivamente útiles para discriminar adecuadamente entre pacientes con cáncer prostático y enfermedad benigna, especialmente cuando el tacto rectal es negativo.

PALABRAS CLAVE: Antigeno prostático específico. Cáncer de próstata. Hiperplasia benigna de próstata. Diagnóstico. Biopsia. Ecografia.

\section{ABSTRACT \\ VALUE OF PROSTATE SPECIFIC ANTIGEN DENSITY AND PROSTATE SPECIFIC ANTIGEN DENSITY OF THE TRANSITION ZONE FOR THE DIAGNOSIS OF PROSTATE CANCER}

INTRODUCTION: With serum prostate specific antigen (PSA) levels of 4.1 to $10 \mathrm{ng} / \mathrm{ml}$ a significant number of patients are unnecessary subjected to biopsy. We try to determine if the calculation of prostate specific antigen density (PSAD) and prostate specific antigen density of the transition zone (PSADTZ) improve the capacity to discriminate between non-malignant disease and prostate cancer.

METHODS: A prospective study including 314 males with PSA levels between 4.1 and $10 \mathrm{ng} / \mathrm{ml}$ is reported. Transrectal ultrasonography and prostatic biopsy were performed in all of them and total prostate and transition-zone volumes were calculated. PSA density and PSA density of the transition zone were calculated for each patient. Receiver operating characteristics (ROC) curves for PSA, PSAD and PSADTZ were constructed for all the patients and for those patients with digital rectal examination unsuspicious of malignancy, determining the sensitivity and specificity for several cutoff values.

RESULTS: The area under the curve for both, PSAD and PSADTZ, were greater than for PSA ( $<<0.05)$, without any significant differences between PSADTZ and PSAD. The cutouff value of greatest diagnostic efficiency for PSAD was $0.17 \mathrm{ng} / \mathrm{ml} / \mathrm{cc}(71.4 \%$ sensitivity and $55.7 \%$ specificity), while it was $0.41 \mathrm{ng} / \mathrm{ml} / \mathrm{cc}$ for DPSATZ (70\% sensitivity and $61.5 \%$ specificity). For those cases of normal digital rectal examination, no differences were observed between PSA and PSAD but they were between PSA and PSADTZ. In any event, the area under ROC curves was always less than 0.7 , and, in order to avoid a large number of biopsies (high specificity), a large number of cancers are left without diagnosis (low sensitivity).

CONCLUSIONS: We conclude that PSAD and PSADTZ are not excessively useful for adequately discriminating between patients with prostate cancer and those with non-malignant disease, particularly when digital rectal examination is normal.

KEY WORDS: Prostate-specific antigen. Prostate cancer. Prostatatic hyperplasia. Diagnosis. Biopsy. Ultrasonography. 
$\mathrm{E}^{1}$ antígeno prostático específico sérico (PSA), considerado como el mejor marcador tumoral para el cáncer de próstata, muestra ciertas limitaciones en su potencial diagnóstico, ya que algunos pacientes con prostatitis e hiperplasia benigna de próstata (HBP) también pueden presentar niveles elevados de PSA ${ }^{1,2}$. Así pues, pese a tener especificidad de órgano, el PSA no es específico de cáncer y aunque la concentración es significativamente mayor en los hombres con cáncer prostático confinado al órgano que en los pacientes con HBP, se ha mostrado un solapamiento de los valores entre ambos ${ }^{3}$. El principal problema está cuando el rango de PSA está entre 4,1 y $10 \mathrm{ng} / \mathrm{ml}$ por lo que se ha establecido una serie de pruebas para mejorar su especificidad, incluyendo el PSA estratificado por edad ${ }^{4}$, la velocidad del PSA ${ }^{5}$, la densidad de PSA ${ }^{6}$, los cocientes PSA libre:PSA total $^{7}$ y complejos PSA:PSA total $^{8}$. Sin embargo, ninguna de ellas es totalmente satisfactoria.

La densidad del PSA (DPSA), se mostró en un principio como un parámetro bastante aceptable para diferenciar carcinoma de próstata de enfermedad benigna ${ }^{9,10}$, aunque estudios posteriores presentaron resultados dispares ${ }^{11,12}$.

Por otra parte, el PSA es secretado principalmente por las células epiteliales prostáticas. La mayor parte del PSA que difunde de la próstata a la sangre proviene de la zona transicional ${ }^{13}$ y el crecimiento prostático se produce, la mayoría de las veces, por crecimiento de esta zona, por lo que el uso de la densidad del PSA de la zona de transición (DPSAZT) se ha sugerido que puede mejorar la especificidad para la detección de cáncer prostático en pacientes con niveles intermedios de PSA $^{14}$.

Nuestro objetivo es determinar si el cálculo de la DPSAZT, en un grupo de pacientes con cifras de PSA entre 4 y $10 \mathrm{ng} / \mathrm{ml}$, mejora la capacidad del PSA y de la DPSA para discriminar entre enfermedad benigna y cáncer y así poder evitar la realización de biopsias innecesarias sin que se vea afectada la capacidad diagnóstica.

\section{PACIENTES Y MÉTODO}

Entre febrero de 1998 y diciembre de 2001 hemos realizado 314 biopsias prostáticas transrectales en pacientes con PSA entre 4,1 y 10 $\mathrm{ng} / \mathrm{ml}$. Los pacientes fueron remitidos de la consulta de urología y no representan un screening poblacional. Para la determinación sérica del PSA, que se realizó previamente a la realización de la biopsia prostática, se utilizó un test de inmunoanálisis (Chemiluminescent Microparticle Immunoassay) de laboratorios Abbott. Se realizó un tacto rectal antes de practicar la ecografía transrectal definiendo los hallazgos como sugestivos de malignidad o no. La ecografía transrectal la realizamos con un ecógrafo Siemens Sonoline SI-200 con sonda endorrectal poliplanar a $7.5 \mathrm{MHz}$. El paciente se coloca en decúbito lateral izquierdo. Se realizó profilaxis antibiótica con ciprofloxacino y preparación rectal con un enema cassen de 250 cc 1 hora antes del procedimiento. Se realizó estudio ecográfico transrectal de la próstata delimitando áreas sospechosas de malignidad, limites prostáticos y vesículas seminales antes de la biopsia. El volumen prostático total y de la zona transicional se calculó utilizando la fórmula del elipsoide con los 3 diámetros axiales prostáticos y de la zona de transición (anteroposterior $\mathrm{x}$ transversal $x$ longitudinal x 0,52). La DPSA se calculó dividiendo la cifra de PSA por el volumen prostático. La DPSAZT se determinó dividiendo la cifra de PSA por el volumen de la zona transicional. Se realizó biopsia en sextante utilizando una pistola automática con aguja tipo tru-cut de 18 G. Biopsias extraordinarias fueron realizadas cuando se detectaron zonas sospechosas con la ecografía. Las muestras obtenidas fueron examinadas para descartar la presencia de cáncer. La neoplasia intraepitelial prostática no fue considerada como lesión maligna.

Estudio estadístico: Se compararon las medias de PSA, DPSA y DPSAZT de los pacientes con cáncer y con enfermedad benigna mediante la $t$ de Student. Para establecer la utilidad de dichos parámetros en la predicción del carcinoma se utilizaron las curvas de rendimiento diagnóstico ("Receiver Operating Characteristic curves" o "ROC curves"), comparando las áreas bajo la curva obtenidas y calculando la sensibilidad y especificidad para diferentes puntos de corte: el de mayor eficiencia diagnóstica, los del 90 y 100\% de sensibilidad, y el valor más representativo de la literatura. El nivel de significación estadística se estableció en el $5 \%$. 


\section{RESULTADOS}

La edad media de los pacientes biopsiados fue de $66( \pm 7)$ años. El PSA medio de la muestra fue de $7,26( \pm 1,60) \mathrm{ng} / \mathrm{ml}$. El 22,3\% (70/314) de las biopsias fueron positivas para carcinoma. El $16,2 \%$ de pacientes $(51 / 314)$ presentaban el tacto rectal sospechoso de malignidad. Entre ellos, el 47,1\% (24/51) se diagnosticaron de carcinoma, Cuando el tacto rectal fue negativo, el $17,5 \%$ de los casos $(46 / 263)$ fueron carcinoma. El volumen medio de las próstatas de la muestra fue de 46 cc y el volumen medio de la zona transicional fue de $22 \mathrm{cc}$.

En la Tabla I se muestran las diferencias entre las medias en el grupo de pacientes con carcinoma y HBP. No observamos diferencias entre las medias del PSA sérico entre los pacientes con cáncer y enfermedad benigna. Tanto la DPSA y la DPSAZT muestran diferencias significativas entre ambos grupos de pacientes.

Para el total de la muestra el área bajo la curva de la DPSA fue mayor que la del PSA solo. A su vez el área bajo la curva de la DPSAZT fue significativamente mayor (Figura 1 y Tabla II). No se observaron diferencias entre DPSA y DPSAZT $(\mathrm{p}>0,05)$.

Cuando analizamos los pacientes con tacto rectal negativo, si bien las áreas bajo la curva son superiores tanto para la DPSA como la DPSAZT, sólo la DPSAZT muestra diferencias significativas (Figura 2 y Tabla II).
En la Tabla II se muestra la sensibilidad y especificidad para los diferentes puntos de corte: el de mayor eficiencia diagnóstica, los que tienen un $90 \%$ de sensibilidad y para los valores de 0,15 $\mathrm{ng} / \mathrm{ml} / \mathrm{cc}$ para la DPSA y 0,35 $\mathrm{ng} / \mathrm{ml} / \mathrm{cc}$ para la DPSAZT.

\section{DISCUSIÓN}

El hecho de que la hipertrofia benigna de próstata (HBP) incrementa los niveles séricos de PSA $^{15}$ ocasionó que se intentara mejorar la sensibilidad y especificidad diagnóstica de este refiriéndolo al volumen prostático, con lo que se introdujo el concepto de DPSA ${ }^{6}$. Posteriormente, mediante análisis discriminante, se calculó la probabilidad de biopsia positiva para cáncer, de tal forma que para pacientes con tacto rectal normal y DPSA menor de $0,15 \mathrm{ng} / \mathrm{ml} / \mathrm{cc}$ no estaría indicada la biopsia, ya que la probabilidad de cáncer sería menor del $6 \%$ y lo adecuado sería establecer un seguimiento con controles periódicos de PSA y tacto rectal ${ }^{16}$.

En un estudio prospectivo, mediante curvas de rendimiento diagnóstico (ROC curves), se determinó 0,15 como valor más adecuado para la indicación de biopsia prostática, con una sensibilidad del $91,3 \%$ y especificidad del $63,0 \%{ }^{17}$, corroborándose estos resultados en estudios posteriores ${ }^{18-20}$. Este valor de la DPSA ha sido el más utilizado para indicar la realización de una biopsia prostática.

\section{TABLA I}

COMPARACIÓN DE MEDIAS DEL PSA SÉRICO, DENSIDAD DE PSA Y DENSIDAD DE PSA PARA LA ZONA TRANSICIONAL EN PACIENTES CON HBP Y CÁNCER DE PRÓSTATA.

\begin{tabular}{|c|c|c|c|}
\hline & HBP & $\begin{array}{l}\text { Cáncer de } \\
\text { próstata }\end{array}$ & $\begin{array}{c}\text { Valor de } p \\
\text { (t de Student) }\end{array}$ \\
\hline \multicolumn{4}{|c|}{ Todos los pacientes } \\
\hline № pacientes (\%) & $244(77,7)$ & $70(22,3)$ & \\
\hline Media PSA & $7,24( \pm 1,62)$ & $7,32( \pm 11,52)$ & N.S \\
\hline Media DPSA & $0,18( \pm 0,09)$ & $0,23( \pm 0,09)$ & $<0,001$ \\
\hline Media DPSAZT & $0,43( \pm 0,29)$ & $0,65( \pm 0,47)$ & $<0,001$ \\
\hline \multicolumn{4}{|c|}{ Tacto rectal normal } \\
\hline № pacientes (\%) & $217(82,5)$ & $46(17,5)$ & \\
\hline Media PSA & $7,27( \pm 1,62)$ & $7,46( \pm 1,46)$ & N.S \\
\hline Media DPSA & $0,17( \pm 0,08)$ & $0,21( \pm 0,08)$ & $<0,01$ \\
\hline Media DPSAZT & $0,42( \pm 0,28)$ & $0,61( \pm 0,39)$ & $<0,01$ \\
\hline
\end{tabular}

N.S.: No significativo 
TABLA II

VALIDEZ DE LOS PARÁMETROS RELACIONADOS CON EL PSA PARA DISCRIMINAR ENTRE CÁNCER DE PRÓSTATA E HIPERPLASIA BENIGNA DE PRÓSTATA SEGÚN EL RESULTADO DE LAS CURVAS DE RENDIMIENTO DIAGNÓSTICO ("ROC CURVES")

\begin{tabular}{|c|c|c|c|c|}
\hline Parámetro & ABC (I.C. 95\%) & Sensibilidad (\%) & Especificidad (\%) & Valor de $\mathbf{P}$ \\
\hline \multicolumn{5}{|l|}{ Todos los pacientes } \\
\hline PSA total $(\mathrm{ng} / \mathrm{ml})$ & $0,518(0,461-0,575)$ & & & \\
\hline $6,8^{1}$ & & 68,6 & 42,2 & \\
\hline $4,9^{2}$ & & 90,0 & 9,0 & \\
\hline $4,1^{3}$ & & 100,0 & 0,0 & \\
\hline DPSA & $0,670(0,615-0,721)$ & & & $<0,01^{4}$ \\
\hline $0,17^{1}$ & & 71,4 & 55,7 & \\
\hline $0,11^{2}$ & & 90,0 & 23,8 & \\
\hline $0,07^{3}$ & & 100,0 & 4,9 & \\
\hline $0,15^{5}$ & & 75,7 & 45,5 & \\
\hline DPSAZT & $0,683(0,629-0,734)$ & & & $<0,01^{4}$ \\
\hline $0,41^{1}$ & & 70,0 & 61,5 & \\
\hline $0,27^{2}$ & & 90 & 31,6 & \\
\hline $0,14^{3}$ & & 100 & 5,7 & \\
\hline $0,35^{5}$ & & 72,9 & 50,4 & \\
\hline \multicolumn{5}{|c|}{ Tacto rectal negativo } \\
\hline PSA total $(\mathrm{ng} / \mathrm{ml})$ & $0,538(0,476-0,599)$ & & & \\
\hline $7,0^{1}$ & & 69,6 & 45,6 & \\
\hline $5,1^{2}$ & & 90 & 11,1 & \\
\hline $4,1^{3}$ & & 100 & 0,0 & \\
\hline DPSA & $0,644(0,582-0,701)$ & & & N.S. ${ }^{4}$ \\
\hline $0,17^{1}$ & & 65,2 & 58,1 & \\
\hline $0,11^{2}$ & & 90 & 24,9 & \\
\hline $0,07^{3}$ & & 100 & 5,1 & \\
\hline $0,15^{5}$ & & 67,4 & 47,5 & \\
\hline DPSAZT & $0,672(0,611-0,728)$ & & & $<0,05^{4}$ \\
\hline $0,42^{1}$ & & 65,2 & 64,5 & \\
\hline $0,25^{2}$ & & 90,0 & 26,3 & \\
\hline $0,14^{3}$ & & 100 & 6,0 & \\
\hline $0,35^{5}$ & & 67,4 & 52,5 & \\
\hline
\end{tabular}

${ }^{1}$ Punto de corte con la mayor eficiencia diagnóstica.

${ }^{2}$ Punto de corte con sensibilidad del 90\%.

${ }^{3}$ Punto de corte con sensibilidad del $100 \%$.

${ }^{4}$ Respecto al PSA total.

${ }^{5}$ Puntos de corte habitualmente preestablecidos en la literatura.

N.S.: No significativo. 
En nuestro estudio, a pesar de que observamos diferencias significativas en la DPSA del grupo de pacientes con cáncer y sin él, estableciendo el límite inferior de PSA en $0,15 \mathrm{ng} / \mathrm{ml} / \mathrm{cc}$ para la indicación de biopsia, evitaríamos realizar un $45 \%$ de estas pero dejaríamos de diagnosticar un $25 \%$ de neoplasias. Igualmente Catalona et al., para el valor de 0,15 , describen una sensibilidad del 59\%, recomendando un punto de corte de 0,078 para conseguir una sensibilidad del $95 \%$ con una especificidad del $19 \%{ }^{21}$. Brawer y cols. no encuentran diferencias estadísticas entre los grupos con biopsia prostática positiva y negativa con valores medios de DPSA de 0,192 y 0,174 para cada grupo respectivamente y sin diferencias en las curvas $\mathrm{ROC}^{12} \mathrm{y}$ al igual que otros estudios $^{22,23}$ no consiguen reafirmar la utilidad de la DPSA, respecto al PSA total, para la detección del cáncer prostático.

La sintesis del PSA depende principalmente de las células del epitelio glandular prostático. La hipertrofia de la zona de transición supone un mayor incremento de las células productoras de PSA por lo que ajustar su nivel plasmático al volumen de la zona de transición puede ser mejor que ajustarlo al volumen prostático total $^{14}$. Zlotta et al muestran que la capacidad para distinguir entre HBP y carcinoma de próstata fue significativamente mayor para la DPSAZT que para la DPSA y que para PSA solo, estableciendo un punto de corte de 0,35 $\mathrm{ng} / \mathrm{ml} / \mathrm{cc}^{24}$. En nuestro estudio observamos que, para toda la muestra, la DPSAZT mejora al PSA solo pero sin que existan diferencias entre DPSA y DPSAZT. Cuando el tacto rectal no es sospechoso de malignidad, aunque la DPSAZT es ligeramente mejor que la DPSA respecto al PSA solo, presenta una baja exactitud (área bajo la curva de 0,67). Así para un punto de corte de $0,35 \mathrm{ng} / \mathrm{ml} / \mathrm{cc}$, sólo diagnosticamos el 67,4\% de los cánceres, evitando el $52 \%$ de biopsias, debiendo disminuirlo a $0,25 \mathrm{ng} / \mathrm{ml} / \mathrm{cc}$ para poder diagnosticar el $90 \%$, pero a expensas de evitar únicamente el 26\% de las biopsias. Maeda et al., mostraron que la DPSAZT puede evitar el $50 \%$ de biopsias, detectando un 25\% menos de tumores con un punto de corte de $0,3 \mathrm{ng} / \mathrm{ml} / \mathrm{cc}$ para valores de PSA entre 4 y $10 \mathrm{ng} / \mathrm{ml}^{25}$. En un estudio prospectivo, para conseguir una sensi- bilidad del $95 \%$ y un $47 \%$ de especificidad se establece el punto de corte en $0,25 \mathrm{ng} / \mathrm{ml} / \mathrm{cc}$, mientras para conseguir esa sensibilidad utilizando la DPSA la especificidad descendía al $21,6 \%$. En este estudio, el punto de corte ideal se establecería en $0,35 \mathrm{ng} / \mathrm{ml} / \mathrm{cc}$, logrando una elevada especificidad (72\%), pero a costa de que la sensibilidad descienda al $79,7 \%{ }^{26}$.

También se ha descrito que la DPSAZT no es mejor que PSAD, aunque es superior al PSA solo $^{27,28}$ En un estudio de screening de cáncer de próstata, DPSA y DPSAZT mostraron un área bajo la curva significativamente mayor que PSA sola, sin que hubiera diferencias entre ellas. El uso de un punto de corte de 0,15 puede evitar un $73,8 \%$ de biopsias pero con un coste de un $43,8 \%$ de cánceres no detectados, mientras que para la DPSAZT un punto de corte de 0,35 evita un $72,9 \%$ pero con un $42,4 \%$ de cánceres no detectados. Con un punto de corte de 0,275 un $60 \%$ de biopsias se evitan pero con pérdida del $28,5 \%$ de cánceres ${ }^{29}$.

Kikuchi et al., mediante un estudio prospectivo, establecen que el área bajo la curva de la DPSAZT es mayor que PSA, DPSA, y las formas moleculares del PSA (PSA libre y PSA-ACT). Con PSA entre 4 y $10 \mathrm{ng} / \mathrm{ml}$, para una sensibilidad del 90\% y especificidad del $58 \%$ se establece un punto de corte de $0,365^{30}$. Defiende este parámetro ya que aunque sea una técnica invasiva si se utiliza en pacientes que acuden a la consulta del urólogo con sintomatología miccional, se realiza una ecografía para diagnosticar la HBP. Sin embargo, en nuestro medio es más habitual realizar una ecografía abdominal que transrectal para el diagnóstico del paciente prostático, reservándose la ecografía transrectal para la realización de biopsia. Así aunque está descrita la correlación entre el volumen prostático calculado mediante ecografía abdominal y el determinado con planimetría transrectal ${ }^{31}$, no es posible medir adecuadamente el volumen de la zona transicional mediante ecografia abdominal. Además, el hecho de que la ecografía urológica, en determinadas circunstancias, la realicen los radiólogos, hace inadecuado el cálculo de la DPSA por la elevada variabilidad que puede existir al medir los diámetros prostáticos.

Moon et al describen que la DPSAZT y cociente PSA libre/total son útiles en la detección del cáncer de próstata en hombres con niveles inter- 


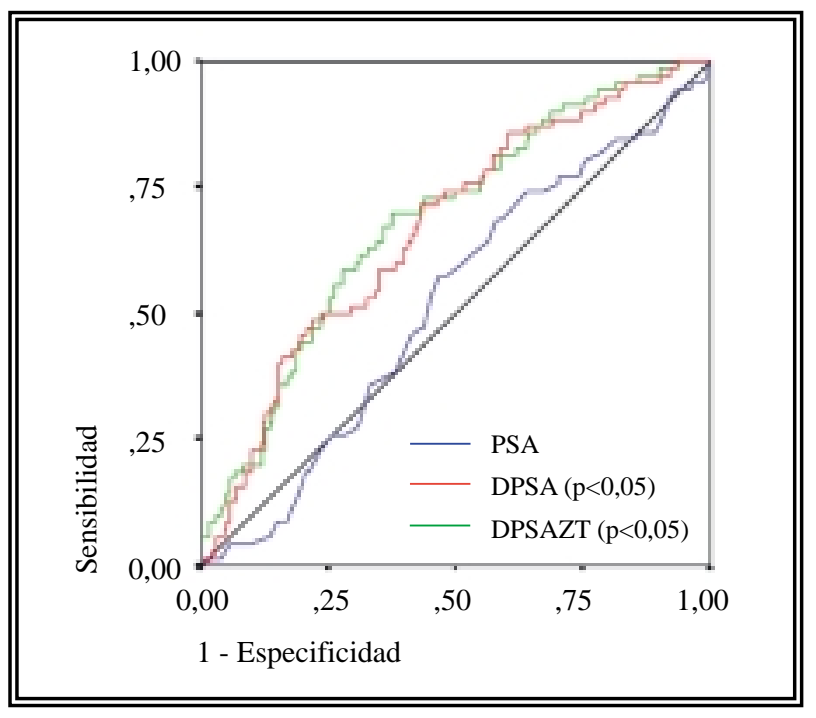

FIGURA 1. Curvas del rendimiento diagnóstico para el PSA, PSA densidad (DPSA) y PSA densidad de la zona de transición (DPSAZT) para toda la muestra.

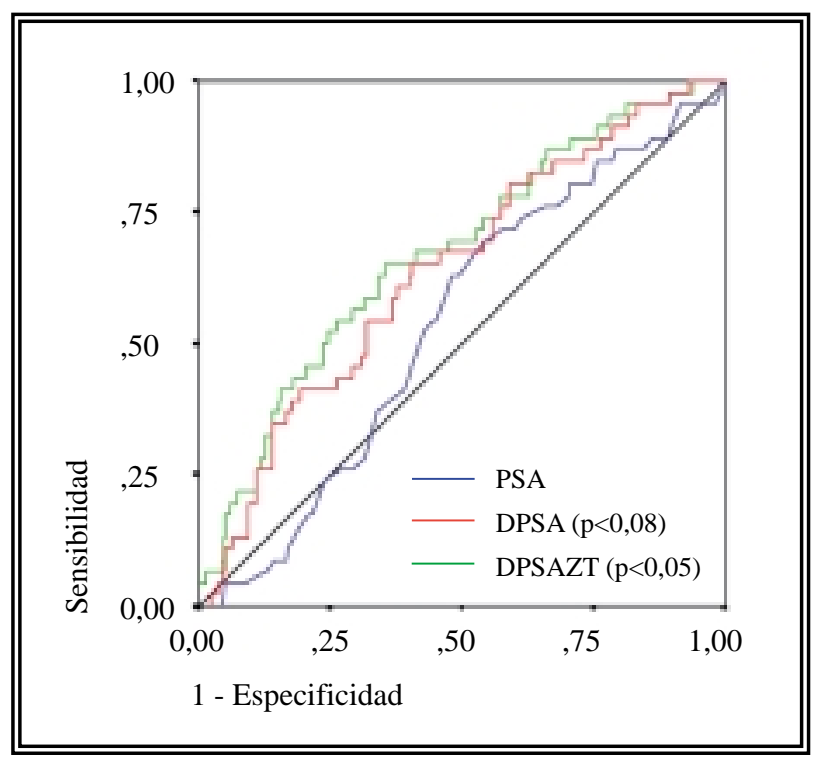

FIGURA 2.Curvas del rendimiento diagnóstico para el PSA, PSA densidad (DPSA) y PSA densidad de la zona de transición (DPSAZT) para los pacientes con tacto rectal negativo.

medios de PSA minimizando el número de biopsias innecesarias. En pacientes con próstatas mayores de $40 \mathrm{ml}$ la DPSAZT es más efectiva para distinguir carcinoma de próstata de HBP. Para la DPSA el valor de 0,15 tiene una sensibilidad del $57 \%$ y una especificidad del $81 \%$. Para un valor de DPSAZT de 0,35 la sensibilidad es del $82 \%$ y la especificidad del $87 \%$. No hay diferencias en el área bajo la curva entre DPSAZT y cociente PSA libre/total ${ }^{32}$. La ventaja del PSA libre es que no se realiza una ecografía transrectal para determinar el volumen prostático y se puede realizar con la misma extracción que la determinación de PSA.

Las discrepancias obtenidas en los estudios se basan en razones de diseño, en razones poblacionales, métodos estadísticos utilizados, así como las diferentes formas de calcular el volumen prostático, ya que no existe un método estandarizado para obtener mediciones lineales de la glándula. Se ha considerado que las diferencias en la especificidad de la DPSA y DPSAZT son probablemente debidas a la aplicación de los mismos puntos de corte a poblaciones de diferente volumen prostático, y que el uso de puntos de corte específicos para cada volumen es más reproducible para la especificidad, de tal forma que próstatas mayores de $30 \mathrm{~g}$ precisan puntos de corte más elevados que las próstatas pequeñas ${ }^{33}$.

Aunque DPSA y DPSAZT mejoren el rendimiento diagnóstico del PSA solo, observamos en nuestro estudio una exactitud relativamente baja de estos parámetros (áreas bajo la curva menores del $0,7)$, tanto en la muestra total como en los pacientes con tacto rectal negativo. Además, cuando consideramos la posibilidad de aplicar la DPSA o la DPSAZT en los programas de detección, la necesidad de realizar una ecografia transrectal en cada caso constituye un serio problema logístico, económico y de incomodidad para el paciente.

Como conclusión, aunque ajustar el PSA al volumen prostático es una práctica común entre los urólogos, observamos que sólo es útil si obviamos la realización de un tacto rectal. Utilizar la DPSAZT aporta escasas ventajas a la DPSA. Hay que tener en cuenta que utilizando la DPSA evitamos la realización de biopsias pero siempre a expensas de dejar sin diagnosticar un número de cánceres. Aunque otros estudios abogan por su uso, incluso en algunos casos puede mejorar el rendimiento diagnóstico del PSA libre, la disparidad de resultados ponen en entredicho su utilidad. La DPSA solamente puede ser utilizado cuando estemos dispuestos a correr el riesgo de que un número de pacientes con cáncer no se diagnostiquen y con la consideración de que, según nuestros resultados y para una sensibilidad del 90\%, es conveniente reducir el umbral de 0,15 a $0,11 \mathrm{ng} / \mathrm{ml} / \mathrm{cc}$. 


\section{REFERENCIAS}

1. OESTERLING JE.: Prostate specific antigen: a critical assessment of the most useful tumor marker for adenocarcinoma of the prostate. J Urol 1991; 145: 907-923.

2. CATALONA WJ, RICHIE JP, AHMANN FR et al.: Comparison of digital rectal examination and serum prostate specific antigen in the early detection and prostate cancer: results o a multicenter clinical trial of 6630 men. J Urol 1994; 151: 1283-1290.

3. BEDUSCHI MC, OESTERLING JE.: Prostate-specific antigen density. Urol Clin North Am 1997; 24: 323-332.

4. OESTERLING JE, JACOBSEN SJ, CHUTE CG, GUESS HA, GIRMAN CJ, PANSER LA, LIEBER MM.: Serum prostate specific antigen in a community-based population of healthy man: establishment of age-specific reference ranges. JAMA 1993; 270: $860-864$.

5. SMITH DS, CATALONA WJ.: Rate of change in serum prostate specific antigen levels as a method for prostate cancer detection. J Urol 1994; 152: 1163-1167.

6. BENSON MC, WHANG IS, OLSSON CA, MCMAHON DJ, COONER WH.: The use of prostate specific antigen density to enhance the predictive value of intermediate levels of serum prostate specific antigen. J Urol 1992; 147: 817-821.

7. CATALONA WJ, SMITH DS, WOLFERT RL, WANG TJ, RITTENHOUSE HG, RATLIFF TL, NADLER RB.: Evaluation of percentage of free serum prostatespecific antigen to improve specificity of prostate cancer screening. JAMA 1995; 274: 1214-1220.

8. MARTÍNEZ SARMIENTO M, ESPAÑA F, ROYO M, ESTELLÉS A, AZNAR J, VERA DONOSO CD, MEDINA P, JIMÉNEZ CRUZ JF.: El cociente PSA:alfa 1 antiquimotripsina/PSA total $(\mathrm{C} / \mathrm{T})$ en el diagnóstico del cáncer de próstata en el rango de PSA total entre 4 y 10 ng/ml. Actas Urol Esp 2001; 25: 269-277.

9. BABAIAN RJ, FRITSCHE HA, EVANS RB.: Prostatespecific antigen and the prostate gland volume: correlation and clinical application. J Clin Lab Anal 1990; 4: 135-137.

10. VENECIANO S, PAVLICA P, QUERZE R, NANNI G, LALANNE MG, VECCHI F.: Correlation between prostate-specific antigen and prostate volume, evaluated by transrectal ultrasonography: usefulness in diagnosis of prostate cancer. Eur Urol 1990; 18: 112-116.

11. LOKNER DH, CRAWFORD ED, DONOHUE RE, MILLER GJ.: Prostate-specific antigen and prostate-specific antigen density in cases of pathologically proven prostate cancer. J Urol 1993; 149: 414A.

12. BRAWER MK, ARAMBURU E, CHEN GL, PRESTON SD, ELLIS WJ.: The inability of prostate-specificantigen index to enhance the predictive value of prostate-specific antigen in the diagnosis of prostatic carcinoma. J Urol 1993; 150: 369-373.

13. HAMMERER PG, MCNEAL JE, STAMEY TA.: Correlation between serum prostate specific antigen levels and the volume of the individual glandular zones of the human prostate. J Urol 1995; 153: 111-114.
14. KALISH J, COONER W, GRAHAM S.: Serum PSA adjusted for volume of transition zone (PSA-T) is more accurate than PSA adjusted for total gland volume (PSAD) in detecting adenocarcinoma of the prostate. Urology 1994; 43: 601-606.

15. NADLER RB, HUMPHREY PA, SMITH DS.: Effect of inflammation and benign prostatic hyperplasia on elevated serum prostate specific antigen levels. $J$ Urol 1995; 154: 407-413.

16. SEAMAN E, WHANG M, OLSSON CA, KATZ A, COONER WH, BENSON MC.: PSA DENSITY (PSAD). Role in patient evaluation and management. Urol Clin North Am 1993; 20: 653-663.

17. BAZINET M, MESHEREF AA, TRUDEL C et al.: Prospective evaluation of prostate specific antigen density and systematic biopsies for early detection of prostatic carcinoma. Urology 1994; 43: 44-51.

18. NISHIYA M, MILLER CJ, LOOKNER DH, CRAWFORD ED.: Prostate specific antigen density in patients with histologically proven prostate carcinoma. Cancer 1994; 74: 3002-3009.

19. ROMMEL FM, AGUSTA VE, BRESLIN JA et al.: The use of prostate specific antigen and prostate specific antigen density in the diagnosis of prostate cancer in a community based urology practice. $J$ Urol 1994; 151: 88-93.

20. WOLFF JM, BOECKMANN W, EFFERT PJ, HANDT S, JAKSE G.: Evaluation of patients with diseases of the prostate using prostate-specific antigen density. Br J Urol 1995; 76: 41-46.

21. CATALONA WJ, SOUTHWICK PC, SLAWIN KM, PARTIN AW, BRAWER MK, FLANIGAN RC, PATEL A, RICHIE JP, WALSH PC, SCARDINO PT, LANGE PH, GASIOR GH, LOVELAND KG, BRAY KR.: Comparison of percent free PSA, PSA density, and age-specific PSA cutoffs for prostate cancer detection and staging. Urology 2000; 56: 255260.

22. AKDAS A, TARCAN T, TURKERI CEVIK I, BIREN T, ILKER Y.: The impact of prostate-specific antigen density in predicting prostate cancer when serum prostate-specific antigen levels are less than 10 ng/ml. Eur Urol 1996; 29: 189-192.

23. COOKSON MS, FLOYD MK, BALL TP Jr, MILLER EK, SAROSDY MF.: The lack of predictive value of prostate specific antigen density in the detection of prostate cancer in patients with normal rectal examinations and intermediate prostate specific antigen levels. J Urol 1995; 154: 1070-1073.

24. ZLOTTA AR, DJAVAN B, MARBERGER M, SCHULMAN CC: Prostate specific antigen density of the transition zone: a new effective parameter for prostate cancer prediction. J Urol 1997; 157: 13151321.

25. MAEDA H, ARAI Y, ISHITOYA S, OKUBO K, AOKI Y, OKADA T.: Prostate specific antigen adjusted for the transition zone volume as an indicator of prostate cancer. J Urol 1997; 158: 2193-2196.

26. DJAVAN B, ZLOTTA AR, BYTTEBIER G, SHARIAT S, OMAR M, SCHULMAN CC, MARBERGER M.: Prostate specific antigen density of the transition zone for early detection of prostate cancer. $J$ Urol 1998; 160: 411-418. 
27. GOHJI K, NOMI M, EGAWA S, MORISUE K, TAKENAKA A, OKAMOTO M, OHORI M, FUJII A.: Detection of prostate carcinoma using prostate specific antigen, its density, and the density of the transition zone in Japanese men with intermediate serum prostate specific antigen concentrations. Cancer 1997; 79: 1969-1976.

28. LIN DW, GOLD MH, RANSOM S, ELLIS WJ, BRAWER MK.: Transition zone prostate specific antigen density: lack of use in prediction of prostatic carcinoma. J Urol 1998; 160: 77-81.

29. RIETBERGEN JBW, KRANSE R, HOEDEMAEKER RF, BOEKEN KRUGER AE, BANGMA CH, KIRKELS WJ, SCHRÖDER FH.: Comparison of prostate-specific antigen corrected for total prostate volume and transition zone volume in a population-based screening study. Urology 1998; 52: 237-246.

30. KIKUCHI E, NAKASHIMA J, ISHIBASHI M, OHIGASHI T, ASAKURA H, TACHIBANA M, MURAI M.: Prostate specific antigen adjusted for transition zone volume: the most powerful method for detecting prostate carcinoma. Cancer 2000; 89: 842849 .
31. MARTÍNEZ JABALOYAS JM, RUIZ CERDÁ JL, OSCA GARCÍA JM, SANZ CHINESTA S, BORONAT TORMO F, JIMÉNEZ CRUZ JF.: Ecografía vesicoprostática versus planimetría transrectal en la determinación del volumen prostático. Actas Urol Esp 1993; 17: 310-314.

32. MOON DG, Y JW, LEE JG, KIM JJ, KOH SK, CHEON J.: The influence of prostate volume on the prostatespecific antigen (PSA) level adjusted for the transition zone volume and free-to-total PSA ratio: a prospective study. BJU International 2000; 86: 670-674.

33. TANEJA SS, TRAN K, LEPOR H.: Volume-specific cutoffs are necessary for reproducible application of prostate-specific antigen density of the transition zone in prostate cancer detection. Urology 2001; 58: 222-279.

Dr. J.Ma Martínez Jabaloyas

Servicio de Urología. Hosp. Clín. Univ. de Valencia Avda. Blasco Ibáñez, 17

46010 Valencia

(Trabajo recibido el 24 marzo de 2003) 